\title{
A MICRO FLUXGATE MAGNETIC SENSOR USING MICROMACHINED 3- DIMENSIONAL PLANAR COILS
}

\author{
Trifon M. Liakopoulos, Ming Xu and Chong H. Ahn
}

\author{
University of Cincinnati \\ Center for Microelectronic Sensors and MEMS (CMSM) \\ Department of Electrical and Computer Engineering and Computer Science \\ P.O. Box 210030, Cincinnati, OH 45221-0030, USA
}

\begin{abstract}
A new micro fluxgate magnetic sensor using micromachined 3-dimensional solenoid coils has been designed, fabricated, and characterized in this work. The fluxgate sensor adopts a ring-type shape and second-harmonic principle, and is composed of exciting coils, a ring core, and sensing coils. Using a newly developed UV lithography-based thick photoresist process and electroplating techniques, planar three-dimensional magnetic fluxgate sensors were fabricated on a silicon wafer. The measured sensor output shows excellent linear characteristics over the range of $\pm 500 \mu \mathrm{T}$ with a resolution of approximately $60 \mathrm{nT}$, achieving the sensitivity of $8360 \mathrm{VT}^{-1}$. Furthermore, this sensor also shows a wide sensing range of $\pm 1.5 \mathrm{mT}$. Using this highly sensitive, on-chip fluxgate sensor, various applications are expected from space research to portable navigation systems.
\end{abstract}

\section{INTRODUCTION}

A wide range of sensing applications requires the detection of magnetic field. Different magnetic sensors and techniques have been developed and are capable of measuring a wide range of DC magnetic fields from $10^{-10}$ to $10^{-3} \mathrm{~T}$.

The fluxgate magnetic sensor [1] is known as one of the most sensitive magnetic sensors. In terms of the range and resolution, the fluxgate sensor stands higher than low sensitivity solid-state devices such as Hall effect and magnetostrictive magnetic sensors, and is comparable to ultrahigh sensitive but very expensive quantum-effect SQUIDs [2].

Since first developed in the 1930s and 1940s for submarine detection, the fluxgate sensors have been used for geophysical prospecting, airborne field mapping, and space applications [3]. They also can serve as orientation sensors for virtual reality applications or as ferromagnetic object detectors. Fluxgate compasses extensively have been part of aircraft and miniaturized ultra sensitive navigation systems.

Recently there has been a large demand for the realization of on-chip fluxgate magnetic sensors, with exciting and sensing control IC circuits. Fabrication of a micro-fluxgate sensor utilizing $\mathrm{Si}$ process technology is driven by advantages such as the small size, light weight, low cost, higher resolution and integration of the supporting electronic circuitry [4-7]. However, when the dimensions of the device decrease, the sensitivity and operation range can have some drawbacks. Some of these can be compensated by increasing the operational frequency, which requires more complex electronic circuits for its signal processing. Micromachined on-chip magnetic sensors can be an applicable solution to address the drawbacks.

In this work we present a micro-fluxgate sensor, with planar three-dimensional solenoid-type coils as excitation and sensing elements. A new planar, UV lithography-based thick photoresist process was developed for the fabrication of the planar solenoid coils [8]. The explored fluxgate sensor is a ring core/second harmonic-type sensor and has a size of $5 \mathrm{~mm} \times 2.5 \mathrm{~mm} \times 100 \mu \mathrm{m}$. The exciting and sensing elements are composed of magnetic cores and solenoid conductor coils, where electroplated $\mathrm{Cu}$ conductors are wound around the electroplated $\mathrm{Ni} / \mathrm{Fe}$ permaloy core. An innovative micro fluxgate magnetic sensor with micromachined planar solenoid-type coils has been designed, fabricated and characterized in this work.

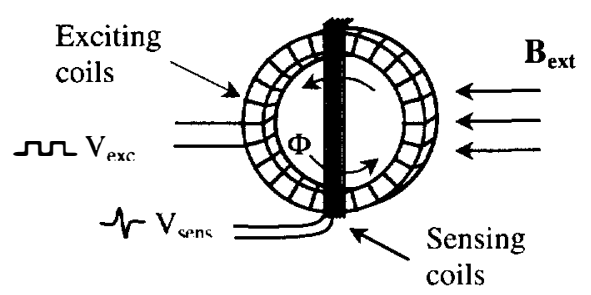

Figure 1. Illustration of a ring core/second harmonic fluxgate magnetometer configuration.

\section{THEORY AND DEVICE DESIGN}

One basic configuration of a fluxgate sensor consists of a magnetic ring core, an excitation coil wound around it, and at least one pickup coil. A schematic illustration of such configuration is shown in Figure 1. A square wave pulse current can be applied to the excitation coils to drive the ring core through its B-H curve. The amplitude of the excitation current is so adjusted that the excitation field oscillates periodically between the positive and negative saturation region. The sensing coils are wound around the whole body of the ring core. The output signal will be proportional to the change of the magnetic flux that flows through the face area of the sensing coils.

Faraday's law describes the induced output voltage at the sensing winding as

$$
V_{e m f}=-N A \mu_{0} \mu_{\mathrm{r}}\left(\frac{d H}{d t}\right)
$$

where $V_{\text {emf }}$ is the induced electromotive force, $N$ is the number of turns, $A$ is the cross section area of the magnetic core, $\mu_{0}$ and $\mu_{r}$ are the magnetic permeabilities of air and magnetic core respectively, and $H$ is the magnetic filed.

If there is no external magnetic field, the closed path of the flux of the excitation field in the core results in zero net flux through the sensing winding. In this case, no output signal in the sensing winding is produced. Whereas, if the core is oscillating, 
with the presence of an external magnetic field $\mathrm{B}_{\mathrm{cxt}}$, from zero magnetization to saturation and backwards (i.e.. twice each excitation period), the flux through the sensing winding changes from a maximum value to zero. The produced output signal will be proportional to the change of the magnetic field flux with time as it is indicated in Equation 1. Since this change is occurring four times for each excitation period, the output pulse has the double frequency with respect to the exciting input signal. Thus, these types of fluxgate sensors are called second harmonic fluxgate magnetometers. Since a square wave input signal produces an abrupt change on the magnetization of the core, a strong output signal can be generated at the sensing coils. Of course, the use of a triangular excitation wave is being adopted in several applications. In the triangular wave case, the output signal will be linearly depended on the excitation frequency, and therefore the sensor is usually driven at higher frequencies to get a high sensitivity.

Based on the configuration described above, we designed a ring-type second harmonic fluxgate sensor as shown in Figure 2. The sensor has a size of $5 \mathrm{~mm} \times 2.5 \mathrm{~mm} \times 100 \mu \mathrm{m}$, where the width of the conductors is $50 \mu \mathrm{m}$ and the spacing between them is $30 \mu \mathrm{m}$. The excitation and pick-up windings are fabricated using electroplated copper. The excitation winding has 41 turns around the magnetic core. The pick up winding has 14 turns around both paths of the magnetic core. As shown in Figure 2(a), the wrapping of the excitation coil around the core is interrupted in the middle of the core, so the pick up coil can be placed in the center. We choose this simple configuration over some more complicated interleaved formation. With this configuration, the pick up winding is more "tight" wound around the core so we can minimize the external noise or coupling interference of the two windings.

(a)
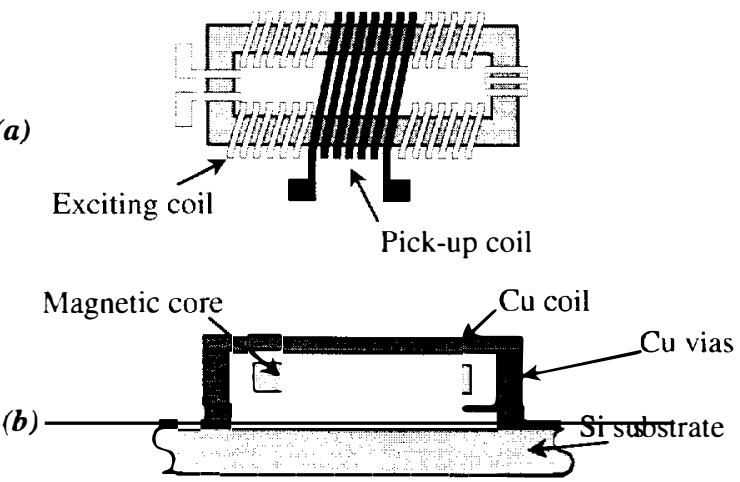

Figure 2. Schematic illustration of a ring type microfabricated magnetic sensor: (a) top view and (b) cut view.

\section{FABRICATION}

The fabrication of the device was based on a newly developed UV lithography based thick photoresist process. The fabrication steps are summarized in Figure 3. We started with a Silicon oxidized wafer on the top of which was deposited $\mathrm{Ti} / \mathrm{Cu}$ seed layer. Using thick photoresist (AZ-4000 series) and conventional UV-lithography, we prepared the molds in the photoresist where the bottom $\mathrm{Cu}$ conductors were to be electroplated. The thickness of the photoresist defines the thickness of the electroplated $\mathrm{Cu}$ conductors. Using electroplating techniques, $25 \mu \mathrm{m}$ thick $\mathrm{Cu}$ was electroplated. The seed layer was then removed and a new photoresist layer was spun to the wafer.

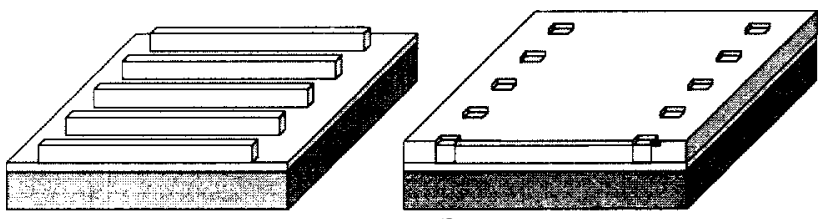

(a)

(b)

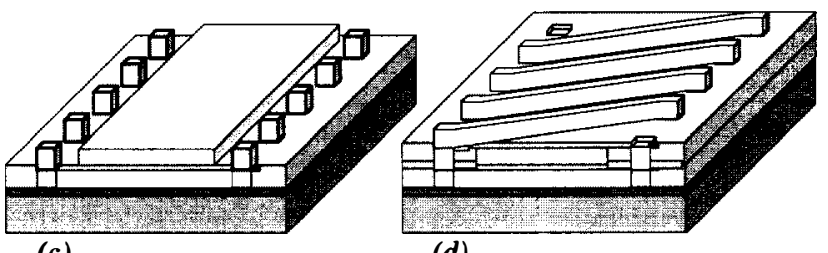

(c)

(d)

Figure 3. UV-lithography and electroplated based fabrication steps: (a) on top of $\mathrm{SiO}_{2}$ wafer, electroplate $\mathrm{Cu}$ conductors; (b) open via connections in hard cured photoresist; (c) electroplate vias and magnetic core; and (d) hard cure the second layer of photoresist and electroplate the top conductors.

After the conductor vias were patterned, a hard curing step was necessary to electrically insulate and support the next layers.

Spinning the photoresist on the top of electroplated $\mathrm{Cu}$ conductors usually gives a non-uniform surface. This can cause problems with the photolithography for the following layers. This problem was solved with a hard curing step. By hard curing at 220 ${ }^{\circ} \mathrm{C}$, we achieved a re-flow of the photoresist and a very smooth surface to continue the fabrication of the next layers. On the top of the hard cured photoresist, we deposited another seed layer, and we patterned and electroplated the vias and then the magnetic core. The conductor vias have a cross section of $50 \mu \mathrm{m} \times 50 \mu \mathrm{m}$ and a thickness of $25 \mu \mathrm{m}$.

The magnetic core is formed from electroplated $\mathrm{Ni} / \mathrm{Fe}$ $(80 \% / 20 \%)$ permalloy. Permalloy is often used as a core material for fluxgate sensors due to its high permeability and fast frequency response. The higher the permeability of the core, the easier saturation can be reached with low excitation current. This is desirable especially when the sensor needs to be integrated with CMOS. The thickness of the electroplated core is very critical in defining the characteristics of the fluxgate sensor. Since the induced output voltage is proportional to the face area of the pickup winding (i.e., total cross-sectional area of the core), the increase of the magnetic core will proportionally improve the sensitivity of the sensor. The advantage of electroplated magnetic core over sputtered one is that the thickness and pattern can be

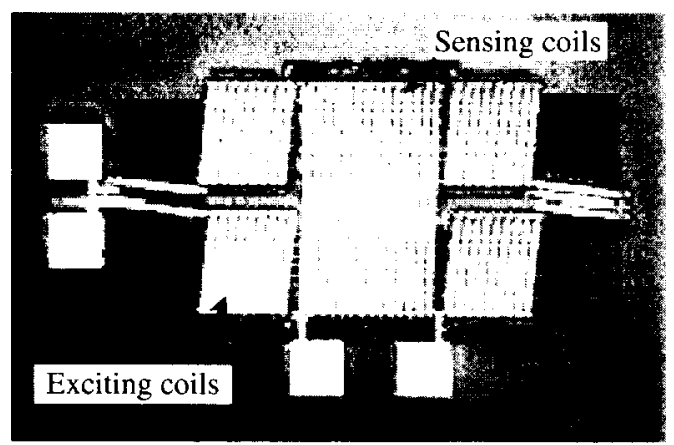

Figure 4. Microphotograph of the fabricated micro-fluxgate sensor with dimensions $5 \mathrm{~mm} \times 2.5 \mathrm{~mm} \times 100 \mu \mathrm{m}$. 
easily controlled to a desirable dimension and shape, so the sensitivity and the operational range of the sensor can also easily be tailored. The devices in this work were fabricated with a $15 \mu \mathrm{m}$ thick magnetic core.

When the vias and magnetic core were in place, we removed the seed layer and we performed another hard curing process. Finally, the last step was followed to pattern and electroplate the $25 \mu \mathrm{m}$ top conductor lines. A microphotograph of the fabricated fluxgate sensor is shown in Figure 4.

\section{EXPERIMENTS}

In order to characterize the fabricated devices, very simple excitation and sensing (input/output) circuits were built. Considering the number of turns of the exciting winding, the geometry of the core, and the saturation magnetization of the electroplated $\mathrm{Ni} / \mathrm{Fe}$ permalloy (e.g., $0.8 \mathrm{~T}$ for the permalloy electroplated in this work), the current that was required to saturate the magnetic core was approximately $220 \mathrm{~mA}$. Figure 5 shows the measurement system of the fluxgate sensor. The driving circuit shown in Figure 5 is able to provide an oscillating current of 300 $\mathrm{mA}$ to ensure saturation of the core. The input of this circuit was connected to a function generator which can provide square or triangular waveforms. A square wave pulse-signal of $3 \mathrm{Vpp}$ was used for these experiments.

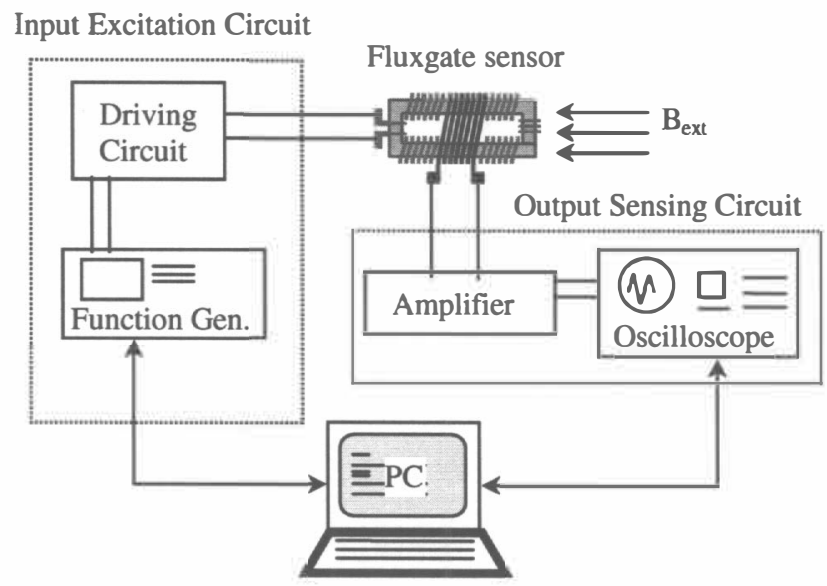

Figure 5. Measurement system of fluxgate sensor

The measured resistance of the exciting coil was $1.2 \mathrm{Ohms}$ over the operating frequencies of $1-200 \mathrm{kHz}$. This low resistance limits the power consumption to approximately $100 \mathrm{~mW}$ in this sensor.

The output signal from the fluxgate sensor needs to be amplified. A commercially available op-amp was used to amplify the output signal approximately 20 times.

The magnetic field to be measured was produced from a solenoid, whose dimensions and electrical and magnetic characteristics are precisely known. In order to measure a wide range of magnetic fields, two different solenoids were used with different number of turns. By precisely controlling the current in the solenoids, we could produce external magnetic fields in the range of $0-1.5 \mathrm{mT}$. The fluxgate sensor was placed in the center of the solenoid, where the magnetic field was assumed homogeneous and constant. A photograph of the fluxgate probe with the electronic circuitry is shown in Figure 6 . The measurements were performed in a magnetically shielded box to

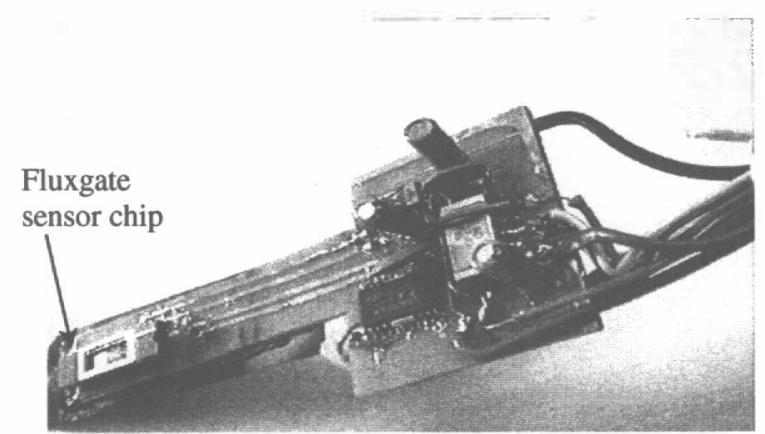

Figure 6. Fluxgate probe with the supporting electronics.

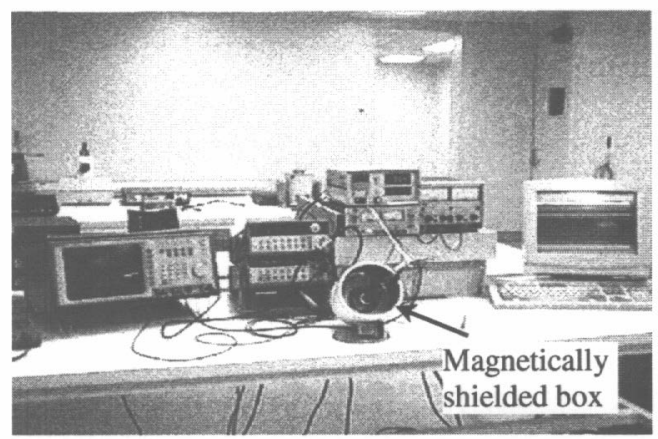

Figure 7. Experimental setup with a magnetically shielded box.

minimize external noises as shown in Figure 7, and the output voltage was measured with a digital oscilloscope.

Since the fabrication process of this sensor can be compatible with CMOS, the supporting driving and sensing electronic circuits can be integrated with the sensor in one chip, adopting a post-fabrication process of the fluxgate sensor.

(a)

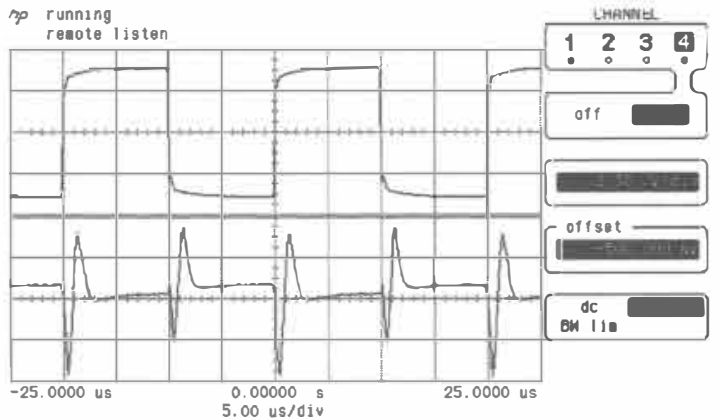

(b)

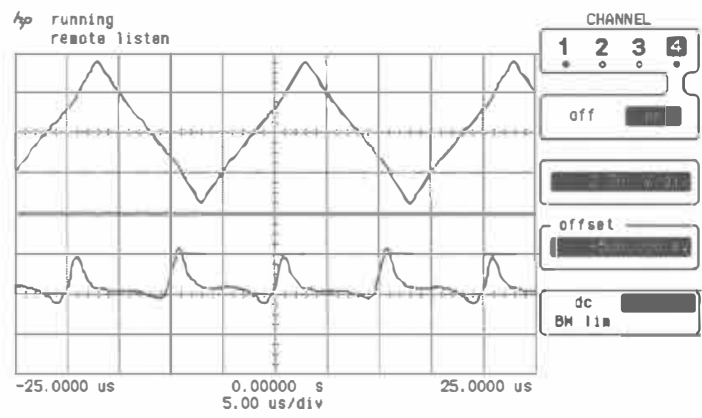

Figure 8. Measured waveform of 3 Vpp input (upper) and output (lower) signal at $50 \mathrm{kHz}$ (a) square wave and (b) triangular wave. 


\section{RESULTS AND DISCUSSION}

In these experiments, we used a square wave input current of $300 \mathrm{~mA}$ to drive the exciting winding. Figure 8 shows the input and output signals of the sensors at a frequency of $50 \mathrm{kHz}$ for the square and triangular input signal, respectively.

The sensitivity of the fluxgate sensor does not have significant dependence on the excitation frequencies when a square wave is used. From Equation 1, the amplitude of the output voltage depends on the change of the magnetic field over time, and thus the square wave is related to the rising and falling time of the pulse. When, a triangular waveform is used, there is a linear dependence of the sensitivity with the excitation frequency. In order to improve the sensitivity, it is preferable to drive the fluxgate sensor at high excitation frequencies with triangular waveforms, but more complicated electronic circuits will be required in this case. In this work however, the thick magnetic core $(\sim 15 \mu \mathrm{m})$ allow us to get fairly high sensitivity at low excitation frequencies with the square wave excitation pulse.

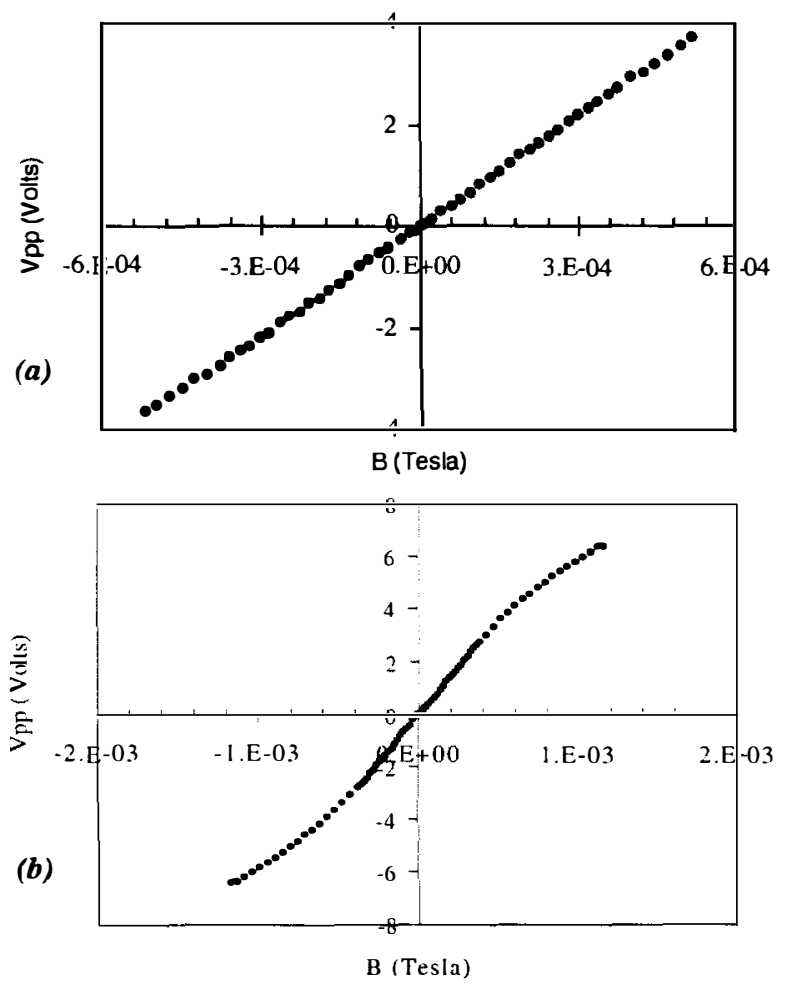

Figure 9. Output signals versus external magnetic field: (a) linear dependence of the amplitude Vpp of the output signal over the external magnetic field $( \pm 500 \mu T)$ and (b) wide range external magnetic field measurements $( \pm 1.3 \mathrm{mT})$.

Measured output signals versus external magnetic fields are plotted in Figure 9. The sensor shows a very linear sensitivity from $-500 \mu \mathrm{T}$ to $+500 \mu \mathrm{T}$ as shown in Figure 9(a). The measured resolution of the sensor is approximately $60 \mathrm{nT}$. The measured sensitivity of the fluxgate sensor cell without any amplification is $360 \mathrm{VT}^{-1}$ for $1 \mathrm{kHz}$ and $418 \mathrm{VT}^{-1}$ for $100 \mathrm{kHz}$. However, the sensitivity of the whole sensor system with amplifiers is approximately $8360 \mathrm{VT}^{-1}$ at $100 \mathrm{kHz}$. To demonstrate a wide sensing range of this sensor, measurements are performed in the range of $-1.3 \mathrm{mT}$ to $+1.3 \mathrm{mT}$ and the results are presented in Figure 9(b). As shown in Figure 9(b), the linearity of the curve begins to break at the magnetic fields over approximately $\pm 500 \mu \mathrm{T}$. From the plots shown in Figure 9, no hysteresis was observed when the measurements were performed in both directions.

The geometry and magnetic of the magnetic core is considered as very important factors to shape the performance of the fluxgate sensor. By an optimized thickness of the core, the operational range of the sensor and its sensitivity can be flexibly controlled in this device.

Furthermore, to enhance the sensitivity of the sensor with a high frequency excitation, measurements are under development while the frequencies of the triangular excitation pulse are varied.

\section{CONCLUSION}

In this work, a micro fluxgate magnetic sensor has been designed, fabricated and functionally characterized. Micromachining techniques were used to fabricate the fluxgate sensor cell, which is composed of a magnetic core and the 3dimensional planar solenoid coils for the excitation and sensing elements. The sensor cell sensitivity of approximately $400 \mathrm{VT}^{-1}$ without amplification, was achieved over the frequency range of 1 $\mathrm{kHz}-100 \mathrm{kHz}$, but the achieved sensitivity of the whole sensor system was $8360 \mathrm{VT}^{-1}$ at $100 \mathrm{kHz}$. A linear dependence of the output $V_{p p}$ signal was shown for the range of $-500 \mu \mathrm{T}$ to $+500 \mu \mathrm{T}$ with a resolution of approximately $60 \mathrm{nT}$. The power consumption was $\sim 100 \mathrm{~mW}$. Using the highly sensitive, on-chip fluxgate sensor, realized in this work, numerous applications are expected from space research and submarine detection to potable navigation systems and biomedical image systems.

\section{AKNOWLEDGMENTS}

This work was partially supported by a DARPA grant under contract number AF F30602-97-2-0102. The authors thank Hoechst-Celanese company for the AZ-photoresist donation. We would also like to thank Nihat Okulan, Srinivasan Iyengar, Hyoung-Jin Cho and Dan Sadler, for useful discussions and suggestions.

\section{REFERENCES}

[1] F. Primdahl, "The fluxgate magnetometer", J. Phys. E: Sci. Instrum. Vol. 12, pp. 241-253, 1979.

[2] R. S. Popovic, et al, "The future of magnetic sensors", Sensors and Actuators, A56, pp. 39-55, 1996.

[3] R. Ripka, "Review of fluxgate sensors", Sensors and Actuators, A33, pp. 129-141, 1992.

[4] S.O. Choi, S. Kawahito, et al, "An integrated micro fluxgate magnetic sensor", Sensors and Actuators, A55, pp. 121-126, 1996.

[5] S. Kawahito, H. Satoh, et al, "High resolution micro-fluxgate sensing elements using closely coupled coil structures", Sensors and Actuators, A54, pp. 612-617, 1996.

[6] R. Gottfried-Gottfried, et al., "A miniaturized magnetic-field sensor system consisting of planar fluxgate sensor and a CMOS readout", Sensors and Actuators, A54, pp. 443-447, 1996.

[7] T. Seitz, "Fluxgate Sensor in Planar Microtechnology", Sensors and Actuators, A21-A23, pp. 799-802, 1996.

[8] T. M. Liakopoulos et all, "A bio-magnetic bead separator on glass chip using semi-encapsulated spiral electromagnets", proc., 1997 International conference on Solid-State Sensors and Actuators, vol.1, pp. 485-488, 1997. 